\title{
Simplified White Blood Cell Differential: An Inexpensive, Smartphone- and Paper-Based Blood Cell Count
}

\author{
Matthew V. Bills, Brandon T. Nguyen, and Jeong-Yeol Yoon
}

\begin{abstract}
Sorting and measuring blood by cell type is extremely valuable clinically and provides physicians with key information for diagnosing many different disease states including: leukemia, autoimmune disorders, bacterial infections, etc. Despite the value, the present methods are unnecessarily costly and inhibitive particularly in resource poor settings, as they require multiple steps of reagent and/or dye additions and subsequent rinsing followed by manual counting using a hemocytometer, or they require a bulky, expensive equipment such as a flow cytometer. While direct on-paper imaging has been considered challenging, paper substrate offers a strong potential to simplify such reagent/dye addition and rinsing. In this work, three-layer paper-based device is developed to automate such reagent/dye addition and rinsing via capillary action, as well as separating white blood cells (WBCs) from whole blood samples. Direct on-paper imaging is demonstrated using a commercial microscope attachment to a smartphone coupled with a blue LED and $500 \mathrm{~nm}$ long pass optical filter. Image analysis is accomplished using an original MATLAB code, to evaluate the total WBC count, as well as differential WBC count, i.e., granulocytes (primarily neutrophils) vs. agranulocytes (primarily lymphocytes). Only a finger-prick of whole blood is required for this assay. The total assay time from finger-prick to data collection is under five minutes. Comparison with a hemocytometry-based manual counting corroborates the accuracy and effectiveness of the proposed method. This approach could be potentially used to help make blood cell counting technologies more readily available, especially in resource poor, point-of-care settings.
\end{abstract}

Index Terms - Acridine Orange, blood count, cell identification, paper microfluidics, smartphone

\section{INTRODUCTION}

$\mathrm{B}$ lood tests are frequently employed to evaluate human health. One of the most straightforward blood tests is to quantify and identify the blood cell types. A complete blood count (CBC) is primarily a measure of these cellular components and is one of the most routinely ordered blood tests by clinicians. CBCs, especially white blood cell (WBC) count, provide physicians with key information valuable for diagnosing many different disease states including: anemia, leukemia, autoimmune disorders, fungal, and bacterial infections as well as a host of other ailments [1], [2].

Currently there are two methods primarily used to obtain a $\mathrm{CBC}$, specifically a WBC count. The first requires a clinician

Manuscript received March 19, 2019. This work was supported by the Biomedical Imaging and Spectroscopy Training Grant from the U.S. National Institutes of Health, grant number T32-EB000809. Corresponding author: Jeong-Yeol Yoon. or trained lab specialist to prepare blood smear slides, stain them, and then manually count different WBC types using a hemocytometer under a microscope [3]. To do this they must dilute specimens in a red blood cell (RBC) lysing solution to remove RBCs and count WBCs. Manually counting WBCs is laborious and requires specialized medical equipment and trained personnel. The second method employs a flow cytometer, an extremely bulky and expensive piece of equipment, to perform the cell count [4]. This method requires treatment of the whole blood sample using several reagents; typically, RBC lysing solutions and costly antibodies. However, this technique has an extremely high efficiency in accurately identifying and quantifying different WBC subtypes.

Despite their values, the cost and complexity of flow cytometers and traditional cell counting methods in pathology laboratories are inhibitive. This is particularly true in resource poor settings where the benefit of these lab tests is most lacking. Point of care (POC) lab testing is medical screening that can be performed rapidly, and often more affordably either individually or in consultation with a clinician. POC tests' greatest strength is in their ability to provide clinicians and patients with immediate results to help make informed decisions about treatment [5]. POC tests are typically straightforward devices which can be used in ambulatory and resource poor settings with minimal training.

Numerous POC tests have been demonstrated for CBC analysis, typically employing silicone-based microfluidic platforms coupled with optical or electrical detection [6]-[9]. Many of these platforms are powerful and provide accurate and complete CBCs, and a few them have been commercialized. These models fall under two major categories: 1) Hemocytometer-like platforms, creating a monolayer of cells. Using this approach sub-types of WBCs (e.g. neutrophils, lymphocytes, etc.) can be identified by employing fluorescent dyes like Acridine Orange or CD-targeting antibodies [6], [7]. 2) Flow cytometer-like approaches, sorting cells using various flow dynamics ranging from centripetal flow dynamics to size exclusion [8], [10]. Detection using these approaches often employ fluorescence and/or dynamic light scattering.

On the other hand, very few platforms have been demonstrated using a paper based microfluidic platform. In one of the rare exceptions, Zhang et al. employed paper coupled

M.V. Bills, B.T. Nguyen, and J.-Y. Yoon are with the Department of

Biomedical Engineering, The University of Arizona, Tucson, AZ 85721, USA (e-mail: billsm@email.arizona.edu, btnguyen@email.arizona.edu, and jyyoon@email.arizona.edu). 
with gold nanoparticles to detect and measure total WBC concentrations [11]. In another relevant work by Wang et al. [12], a high precision WBC count detection was demonstrated on paper. However, their work required cross-validation with pure WBC samples with known concentrations. More importantly their blood screen is not a WBC differential, i.e., identification of WBC sub-types.

Paper substrates offer two major benefits over the popularlyused silicone-based microfluidic platforms: 1) its ability to filter out blood cells from plasma, or even isolating WBCs from RBCs without using lysing solution, and 2) spontaneous flow by capillary action, dismissing the requirement of external pumping [13], [14], [15], [16]. However, to the best of our knowledge, no work has been demonstrated to utilize these advantages of paper-based microfluidic platforms toward cell sorting and quantification of WBC components.

In this work, we designed, fabricated, and demonstrated a smartphone-based, paper microfluidic platform that could separate RBCs and count WBCs and their sub-types (granulocytes vs. agranulocytes) from small blood volumes (as little as 1-4 $\mu \mathrm{L}$ of whole blood) without using RBC lysing solution or antibodies. Over the course of this project we explored and tested many chip layouts, geometries, paper substrates, and fluorescent probes. We settled on a stacked paper microfluidic platform consisting of three separate paper types: 1) large pore fiberglass, 2) a fine powdered borosilicate filtration membrane, and 3) cellulose chromatography paper. This platform separates cells based on microfiltration and is compatible with fluorescence microscopy detection either employing an inexpensive, commercially available smartphone microscope attachment, optical filter, and an LED attached to a battery; or using a benchtop fluorescence microscope. Using this method, we have been able to demonstrate a clear separation of total WBCs from other cellular components and been able to measure the total WBC count as well as the relative ratios of granular and agranular WBCs.

While there are several different types of WBCs, the two most common types are neutrophils (35-80\% of WBCs) and lymphocytes (18-45\% of WBCs). Neutrophils make up the major portion of granulocytes (so called because they have the visible granules within them), while lymphocytes make up the major portion of agranulocytes (lacking visible granules). Neutrophils and lymphocytes account for roughly $90 \%$ of WBCs found in blood [17]. Therefore, determining the granulocyte count and agranulocyte count can provide clinicians with a reasonably accurate estimate of neutrophil and lymphocyte concentrations. Acridine orange (AO) has been demonstrated to be a useful stain to differentially count granulocytes and agranulocytes [18], [19]. While AO is primarily a green fluorescent nucleic acid dye, it also changes its fluorescence based on the $\mathrm{pH}$. AO is taken up in lysosomes and granules that typically have notably higher $\mathrm{pHs}$ than the rest of a cell. Granulocytes (as the name would suggest) have many granules and as a result have a red shift in their fluorescence. Separating the image into red and green channels and measuring the relative intensities allows us to determine the ratio of granulocytes and agranulocytes in the blood.

This three-layered paper microfluidic device effectively separates WBCs from whole blood, which provides a concentration dependent readout of WBC counts. Additional
AO staining also provides a readout of the ratio of granulocytes and agranulocytes in the blood. Coupled with smartphone optical detection this approach demonstrates an approach to make blood cell counting technologies more readily available, especially in resource poor, point-of-care (POC) settings. This method offers a number of benefits: First, WBCs are able to be concentrated on the platform from whole blood improving the accuracy of the test without relying on centrifuges, other laboratory equipment, or RBC lysing solution. In addition, working with paper significantly reduces the cost, rendering the method disposable. Additionally, individual chips are prepared in advance, including preloading fluorescent dyes (AO) onto the platform prior to use. The platform only requires $1-4 \mu \mathrm{L}$ of blood per test, allowing to be used for finger-prick samples.

\section{Methodology}

\section{A. Paper Microfluidic Device Layout and Fabrication}

The paper-based device consists of three layers of paper and porous membrane stacked together, each with a specific role and function and properties to match to create separation of whole blood constituents.

Layer 1 is a thin sheet of fiberglass (GFCP203000; EMD Millipore Corp., Burlington, MA, USA) which consists of large overlapping glass fibers, with visible pores (pore size $>50 \mu \mathrm{m}$ ). It is preloaded with $\mathrm{AO}$, which is a $\mathrm{pH}$ dependent, cell permeant nucleic acid binding fluorescent dye. The AO is allowed to dry prior to assembly with the other layers. AO's differentiation of granulocytes from agranulocytes was explained briefly in the Introduction. The role of the top layer is two-fold: first, treat the biological specimen with fluorescent dye (AO in this case) that will be used to detect the cells; additionally, the top layer forces the blood specimen to spread out resulting in a more even layer of WBCs at the underlying capture interface (layer 2).

In paper microfluidics, channels are typically produced by creating a hydrophobic barrier around where the fluid should flow. This is typically done either by lithography or by wax printing. Since both methods were not applicable to fiberglass, we utilized paraffin wax film (PM992/EMD; Bemis Company Inc., Neenah, WI, USA). A $20 \mathrm{~mm}$ x $10 \mathrm{~mm}$ square of $1 \mathrm{~mm}$ thick paraffin wax film was folded in half, resulting in a $10 \mathrm{~mm}$ $\mathrm{x} 10 \mathrm{~mm}$ square, and a $6 \mathrm{~mm}$ diameter hole was punched in the center of it. The paraffin film was unfolded and then folded around a $10 \mathrm{~mm} \times 10 \mathrm{~mm}$ square of fiberglass to wrap it around. It was then compressed and placed on a hot plate at $135^{\circ} \mathrm{C}$ to allow the wax to melt and bind to the fiberglass, creating a hydrophobic barrier as shown in Figure 1 (black color). Once cooled to room temperature, the fiberglass was preloaded in a darkroom with $10 \mu \mathrm{L}$ of $5 \%$ diluted $\mathrm{AO}$ and stored in a dark dry place.

Layer 2 consists of a 6-mm diameter circle punched out from Whatman GF-D borosilicate membrane (pore size $2.7 \mu \mathrm{m}$; 1823-010; GE Healthcare Bio-Sciences, Pittsburg, PA, USA). The borosilicate membrane's compact powdery material does not exhibit any fluorescent properties or have an affinity to fluorescent dyes. This membrane has been used to capture most WBCs on the surface while allowing RBCs, platelets, and plasma components to pass through. When using whole blood samples of $>40 \%$ dilution, this membrane can become plugged; however, with $\leq 20 \%$ dilution the membrane effectively 
captures most WBCs at the surface, although greater percentage of WBCs become trapped at the center at higher dilutions.

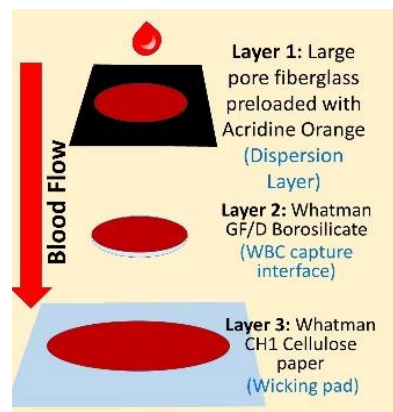

Chip Layout

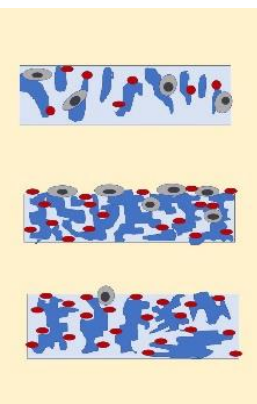

Cross-section

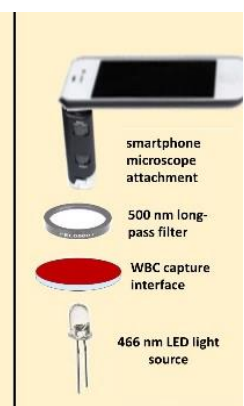

Smartphone Detection
Fig. 1. Device layout: A) three-layered paper microfluidic device design. Layer 1: fiberglass (pore size $>50 \mu \mathrm{m}$ ), preloaded with Acridine Orange (AO), disperses an applied blood sample to provide an even coating of whole blood to the layer 2 (capture interface). Layer 2: Whatman GF/D borosilicate (pore size $2.7 \mu \mathrm{m})$ effectively captures the vast majority of WBCs. Layer 3: Whatman $\mathrm{CH} 1$ cellulose (pore size $11 \mu \mathrm{m}$ ) wicking pad pulls the remaining blood (RBCs and plasma) through the device by capillary action. B) Smartphone imaging platform. The sample is illuminated using a $466 \mathrm{~nm}$ LED light, fluorescence from the sample is filtered through a $500 \mathrm{~nm}$ long-pass filter and magnified using a commercial microscope attachment to a smartphone.

Layer 3 is made up solely of a $20 \mathrm{~mm}$ x $20 \mathrm{~mm}$ sheet of Whatman $\mathrm{CH} 1$ cellulose chromatography paper (pore size 11 $\mu \mathrm{m}$; 1001-813; GE Healthcare Bio-Sciences). The purpose of this sheet is to wick the filtered whole blood samples (RBCs, platelets, and plasma) or phosphate buffered saline (PBS; applied during wash steps), eliminating the need for an external pump or pumping mechanism.

Prior to use with blood samples, the three layers were assembled by applying double-sided tape to the wax-coated edges of the top layer. After that, layer 2 was placed under layer 1 with no overlap with the wax-coated edge where double-sided tape was applied. Layer 3 was then adhered to layer 1 via the double-sided tape. This arrangement was demonstrated to be effective and tightly held all layers closely together. Although wetting the tape did compromise the adhesive, this was beneficial because the assay was typically complete by then and the fluid improved the disassembly of layer 2 for analysis.

\section{B. Sample and Reagent Preparation}

Blood specimen were collected in $10 \mathrm{~mL}$ vials of sodiumheparin or sodium citrate in accordance with the Institutional Review Board (IRB)-approved protocol (approval number 1612094853) from six healthy volunteers ranging in age from 20-35. Samples were stored at $4-6^{\circ} \mathrm{C}$ and used within 48 hours after collection. Prior to use, samples were diluted in PBS ( $\mathrm{pH}$ 7.4) to either $5 \% 10 \%$, or $20 \%$ whole blood by volume. As described in the previous section, high dilutions result in plugging leading to a decreased flow rate and increased assay time. Therefore, $20 \%$ dilution was used as the highest dilution for blood samples with reasonable assay time $(<5 \mathrm{~min})$ and minimum overlapping of WBC images.

A 5\% Acridine Orange solution was diluted from a 10 $\mathrm{mg} / \mathrm{mL}$ stock solution (A8097; Sigma-Aldrich, St. Louis, MO, USA) to differentiate granulocytes vs. agranulocytes on the fabricated device.

\section{Assay Protocol}

Prepared devices were loaded with $20 \mu \mathrm{L}$ of a diluted whole blood sample. After 5 minutes the devices were washed twice by loading $20 \mu \mathrm{L}$ of $1 \mathrm{X}$ PBS to wash excess RBCs, plasma, and AO dye. The cell capture interface (i.e. layer 2) was then separated for fluorescence imaging.

\section{Imaging}

Benchtop microscopic imaging was accomplished by placing the cell capture interface (layer 2) on a glass slide and imaged using a 10X objective and a filter cube for blue bandpass filter excitation and green long-pass filter emission. Nikon TS100 fluorescence microscope (Tokyo, Japan) was used for imaging.

Smartphone-based microscopic imaging was accomplished using a smartphone (iPhone 8; Apple Inc., Cupertino, CA, USA) connected to a commercially available microscope attachment (XFox Professional 300X Optical Glass Lenses; $\mathrm{X} \& Y$ Ind., Shenzhen, China), attached to a $500 \mathrm{~nm}$ long-pass optical filter (FEL0500, Thorlabs Inc., Newton, NJ, USA). Again, smartphone imaging was also accomplished by sandwiching the WBC capture interface between the optical filter and a transparent glass surface. During detection the WBC capture interface is illuminated using a $466 \mathrm{~nm}$ LED (TLHB5100; Vishay, Malvern, PA, USA) powered by a $9 \mathrm{~V}$ battery. The current is limited using a $500 \Omega$ resistor.

\section{E. Data Processing}

Total WBC count: Obtained images were processed using a MATLAB code to identify and count cells. Raw images were split into red and green channels and the higher intensity green channels were selected. Cells were then counted using the built in 'imfindcircles' function with 'twostage' detection to identify cells by finding high intensity regions of appropriate size and circularity. Appropriate properties for the imfindcircles function from the image processing toolbox - gradient threshold and sensitivity - were determined for each dilution by manually adjusting the parameters with the first sample until optimal conditions were obtained.

WBC differential: To measure the red shift in the granulocyte cell population (which to the naked eye is not readily observed), captured images were processed using an original MATLAB code. WBC images were split into red and green color channels. WBCs identified using our total WBC count code were marked with circles and a mask was created to mark all identified circles. The red and green channels were converted to the matrices of the pixel intensities, $\mathrm{x}$ - and $\mathrm{y}$-coordinates at double precision values and scaled up by an addition 10 , so that the pixel values would not be divided by zero at any location. A pixel by pixel ratio of red to green $(\mathrm{R} / \mathrm{G})$ for each of the identified WBC locations was obtained by doing a cell by cell division of the red matrix by the green matrix. The mean intensity at each of the mask locations overlaid onto the red/green (R/G) ratio image was measured using the 'regionprops' function from the image processing toolbox. The mean intensities from the $R / G$ ratios for each cell were converted from a structure to a double precision matrix and plotted as a histogram of frequency vs. R/G color ratio containing 20 bins. This histogram was used to identify two discrete cell populations, granulocytes (mostly neutrophils) and 
agranulocytes (mostly lymphocytes). This was done by manually visualizing the bimodal distribution of the two cell populations and finding the value that most closely corresponds to the cutoff between granulocytes and agranulocytes (often visualized either as a minimum value between the two peaks or occasionally as a small localized third peak where the two populations overlapped) and manually inputting the value into the script. The granulocyte count was then found by finding and counting all cells that had a higher $\mathrm{R} / \mathrm{G}$ ratio than the manually determined R/G cutoff value (Fig. 2A).

\section{F. Comparison with Manual Counts}

To compare our method with a gold standard technique, WBCs were manually counted by a series of reagent/dye additions and rinsing, followed by a hemocytometry counting. WBC counting was performed by making a blood smear on a clean glass slide and allowing it to dry. The slide was dipped into a tube of $2 \%(\mathrm{v} / \mathrm{v})$ acetic acid diluted in deionized (DI) water from 99.67\% stock glacial acetic acid (695092-2.5L-GL; Sigma-Aldrich, St. Louis, MO, USA) to lyse RBCs, followed by washing under running water. Majority of RBCs were removed with this method, although not completely removed. WBCs were then immediately identified and counted using a hemocytometer in a light microscope (Nikon TS100).

Manual differentiation of WBCs was performed using Wright-Geimsa dye, again by a series of reagent/dye additions and rinsing followed by a hemocytometry counting. It was prepared as two field stains: Field stain A was prepared by taking 160 mg Methylene Blue (M1940; Sigma-Aldrich), 100 mg Azure II (861065; Sigma-Aldrich), $1.0 \mathrm{~g}$ disodium dihydrogen phosphate anhydrous (RES20908; Sigma-Aldrich), and $1.25 \mathrm{~g}$ potassium dihydrogen phosphate anhydrous (5.43841; Sigma-Aldrich), dissolved in $100 \mathrm{~mL}$ DI water. Field stain B was prepared by taking $200 \mathrm{mg}$ Eosin-yellow (230251; Sigma-Aldrich), $1 \mathrm{~g}$ disodium dihydrogen phosphate anhydrous and $1.25 \mathrm{~g}$ potassium dihydrogen phosphate anhydrous dissolved in $100 \mathrm{~mL}$ DI water. The samples were made into a blood smear on a clean glass slide and allowed to dry. The slide was dipped into a tube of methanol for 1 minute to fix the cells and air dried. It was then dipped into the field stain B to stain acidic components for 5-6 seconds and immediately washed under running water. It was then dipped into the field stain A to stain basic components for 10-30 seconds and immediately washed under running water. The slide was then imaged with an 100X oil immersion objective tallying up different WBC types, until 100 different WBC types were counted (Fig. 2B). These numbers are assumed to represent the relative percentages in that blood sample.
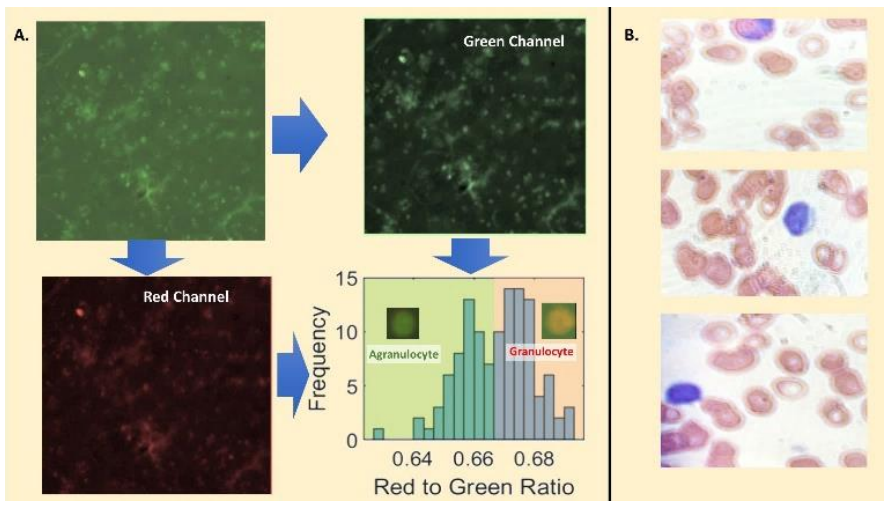

Fig. 2. Granulocyte vs. agranulocyte WBC identification. A) Data processing procedure for granulocyte identification. The obtained image (top left) is split into green $(\mathrm{G})$ and red $(\mathrm{R})$ channel images, processed using a custom MATLAB code to reduce background image noise (top right for the processed green image and bottom left for the processed red image). A histogram of frequency vs. R/G color ratios is created (bottom right) to identify agranulocytes vs. granulocytes. B) Blood smear stained with Geimsa-Wright blood dye used to manually count granulocytes and agranulocytes.

\section{RESULTS AND DISCUSSION}

\section{A. WBC Count Using Benchtop Microscope}

Initial total WBC concentrations (or counts) were evaluated using our microfluidic platform using a benchtop microscope for five different human subjects at three different dilutions $(5 \%, 10 \%$, and $20 \%)$. The input parameters used for the MATLAB function imfindcircles for each dilution on the benchtop microscope are as follows: for the 5\% dilution EdgeThreshold was set to 0.08 , and Sensitivity was set to 0.6 ; for the 10\% dilution EdgeThreshold remained at 0.08 and the Sensitivity was changed to 0.65 ; for the $20 \%$ dilution the EdgeThreshold was set at 0.05 and the Sensitivity remained at 0.65 . Since $20 \mu \mathrm{L}$ of diluted blood sample was loaded to each platform, 1, 2 or $4 \mu \mathrm{L}$ of blood is necessary per test that is comparable to those obtained from a finger prick. For each dilution and each subject, data were captured in $5 \mathrm{X}$ replicates, averaged together, and plotted against the manually counted numbers (i.e., using a series of reagent/dye additions and rinsing followed by a hemocytometry). The results on the benchtop microscope are presented in Figure 3. The curve is divided up by their dilution $(5 \%, 10 \%$, and $20 \%)$. The entire data set could be plotted together on a power curve that demonstrates a clear and predictable correlation between the WBC concentrations using our device and the manual WBC counts. It is interesting to note that higher blood concentrations result in less WBCs being captured at the interface. This can be explained by the fact that the more cells are loaded onto the device results in a greater percentage of them being captured at some other place on the device than at the image plane of the capture layer. This is especially true at very high WBC concentrations where layer 1 begins to get plugged up and allows fewer cells to pass through it. It is also possible that at higher WBC concentrations there is an increased pressure placed on WBCs resulting in a greater percentage of them being absorbed deeper into the layer 2 where they are unable to be differentiated and counted.

This demonstrates that at high dilutions WBCs are retained in very high numbers relative to their concentration. We also 
observe that the borosilicate membrane is effective in retaining WBCs at the imaging focal plane.

Because WBCs are captured depending on whole blood dilution, it becomes meaningful to plot individual dilutions separately so that RBC concentrations and flow dynamics are more similar between samples. $5 \%, 10 \%$, and $20 \%$ whole blood dilutions were separated and analyzed individually and plotted against a linear curve, these dilution-specific linear curves closely demonstrated the most linear trends for the $20 \%$ dilution $\left(\mathrm{R}^{2}=0.9405\right)$ followed by the linear trend of the 5\% dilution $\left(\mathrm{R}^{2}=0.83\right)$. These results demonstrate a strong correlation between the estimated WBC counts using the paper-based device and the manual counting results. This highlights that this device captures cells in a reproducible manner for blood samples diluted between $5 \%$ and $20 \%$. However, the low linearity of the $10 \%$ dilution $\left(\mathrm{R}^{2}=0.53\right)$ should also be recognized. It could likely be improved by obtaining a larger field of view (FOV) because it would reduce the variability between samples. Additionally, the slopes of 5\% and 20\% dilutions are substantially different from each other (0.4123 vs. 0.2342 ), where $5 \%$ dilution covers the lower WBC concentration range and $20 \%$ dilution the higher range. This difference potentially indicates that the curve may not be linear. The 10\% dilution covers the in-between concentration range, and its slope (0.2795) is in between those of 5\% and $20 \%$ dilutions. Its inferior linearity potentially indicates that $10 \%$ dilution samples fall into a transient concentration range.

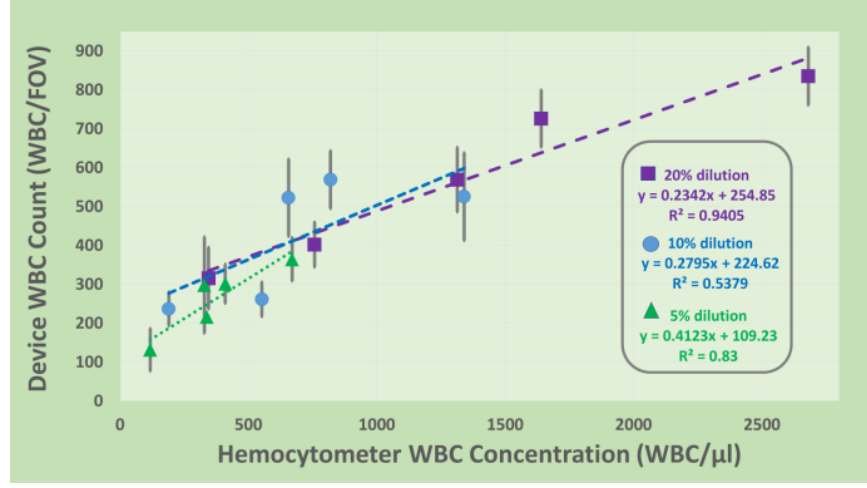

Fig. 3. Total WBC counts per field-of-view (FOV), obtained using the threelayered paper microfluidic device, an original MATLAB code, and a benchtop microscope, are plotted against the manually counted WBC concentrations. All three dilutions were plotted together, each dilution of 5\% (in green triangles), $10 \%$ (in blue circles), or $20 \%$ (in purple squares) were individually fit with a linear curve.

\section{B. Differential WBC Count Using Benchtop Microscope}

A differential WBC count was obtained by staining captured WBC populations with $\mathrm{AO}$, a $\mathrm{pH}$ sensitive nucleic acid stain which exhibits a red shift in acidic environments such as granules. As granulocytes have more lysosomes and granules, they exhibit greater red fluorescence, which can be used to identify and quantify granulocytes.

Blood samples from six human subjects were filtered and imaged on a benchtop fluorescence microscope at three different dilutions $(5 \%, 10 \%$, and $20 \%)$ in $5 \mathrm{X}$ replicates using the same paper microfluidic device. For comparison purposes, the same blood samples were also smeared on glass slides, fixed, stained with a Geimsa-Wright blood dye, and manually analyzed using a hemocytometer under a microscope. Manually tallying WBC types is a laborious process that requires a laboratory setting and trained personnel capable of visually identifying different WBC types. From these numbers, the percentages of granulocytes and agranulocytes in the blood samples were evaluated using both the paper microfluidic device as well as the manual counting method.

The percent granulocyte results from the paper microfluidic device assays using a benchtop fluorescence microscope (lighter gray color) are shown together with those from manual counts (darker gray color) in Figure 4. Both paper microfluidic device and manual counts are within the expected healthy ranges (dotted square box), which confirms the accuracy of this method for identifying the relative ratios of granulocytes and agranulocytes in the blood.

Excessive AO that did not stain the cells may be able to flow through the layer 2, resulting in an elevated background fluorescence in the layer 2. However, as the R/G ratios were measured, and the histograms clearly showed bimodal distributions, the background fluorescence did not affect the assay results - the percentages of granulocytes vs. agranulocytes (WBC differential).

Overall this approach was shown to be effective for separating WBCs into granulocytes and agranulocytes. This data can be taken a step further to estimate the concentrations of lymphocytes and neutrophils in the blood as those two cell types make up approximately $90 \%$ of the total WBC count: majority of granulocytes are neutrophils and majority of agranulocytes are lymphocytes.

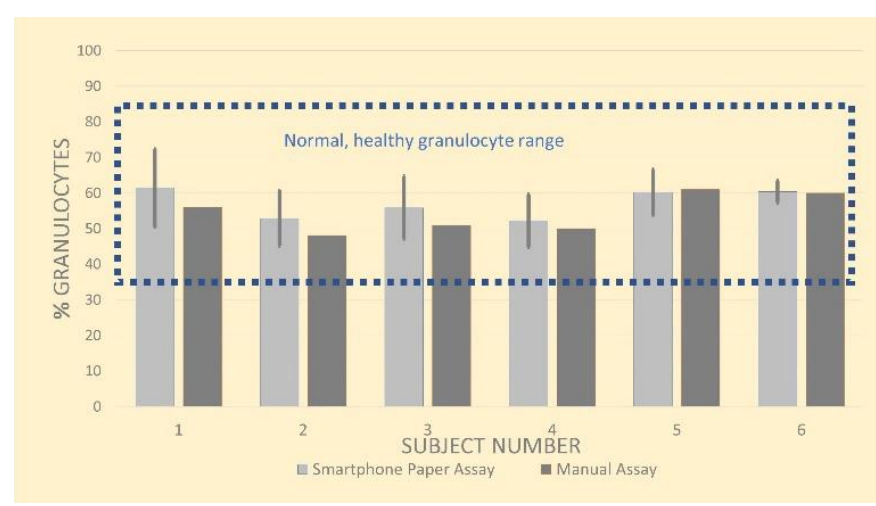

Fig. 4. Differential WBC count: Percent granulocytes for six subjects evaluated by automated, paper microfluidic device assay (light gray), shown together with manual count (dark gray). Error bars represent standard errors for each sample. All results are within the expected healthy range (demonstrated by the dotted square box).

\section{Imaging Using Smartphone Microscope Attachment}

The total WBC concentrations (or counts) were evaluated using our paper microfluidic platform using a smartphone microscope attachment with a $500 \mathrm{~nm}$ long pass optical filter samples for three different human subjects in $3 \mathrm{X}$ replicates. Subjects received a finger prick and $6 \mu \mathrm{L}$ of blood were harvested and diluted to $10 \%(\mathrm{v} / \mathrm{v})$. W BC counts were obtained using a smartphone microscope. Subject WBC concentrations were also manually counted (i.e., using a series of reagent/dye additions and rinsing followed by a hemocytometry) using a 
benchtop microscope. For the MATLAB function imfindcircles for the smartphone microscope data the input value for EdgeThreshold was set to 0.07 and the Sensitivity was set to to 0.7. The results from the smartphone microscope attachment are presented in Figure 5. The entire data set is plotted on a linear trend that demonstrates a clear and predictable correlation $\left(\mathrm{R}^{2}=0.8151\right)$ between the WBC concentrations obtained from our device and by manual counting. These findings show that all three subjects fell within the expected normal WBC concentrations using our device. It is important to note that the field of view (FOV) using the smartphone camera is significantly smaller, leading to substantially smaller WBC counts per FOV than those with a benchtop microscope. However, the results are still able to follow a linear curve for a single dilution and are validated across three patient samples, although, the variability between samples for a subject is higher, which can be attributed to the smaller FOV and/or inferior spatial resolution of a smartphone microscope attachment. It is advisable that three samples should be taken and averaged for each subject.

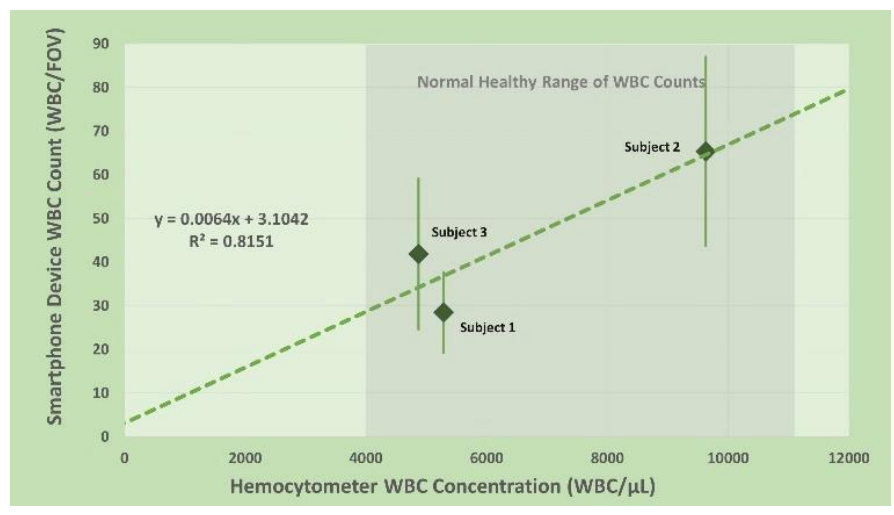

Fig. 5. Total WBC count per FOV with paper microfluidic device using smartphone microscope attachment, compared to the manually counted WBC concentration. Whole blood from three subjects was diluted to $10 \%$. Data was plotted with a linear curve of best fit with an $\mathrm{R}^{2}=0.8151$.

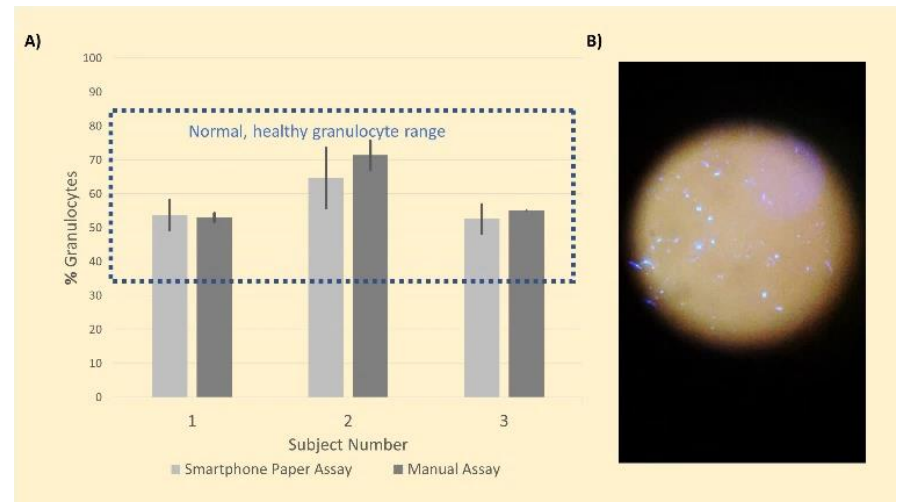

Fig. 6. Differential WBC count using the smartphone-paper system. A) Percent granulocytes for three subjects evaluated by smartphone-based, automated paper microfluidic device assay (light gray), shown together with manual count (dark gray). Error bars represent standard errors for each sample. All results are within the expected healthy range (demonstrated by the blue box). B) Image of WBCs on the capture interface using a smartphone microscope attachment.

A differential WBC count was also obtained using the smartphone-based paper microfluidic device. The percent granulocyte results from this smartphone-paper system (lighter gray color) are shown together with those from manual counts (darker gray color) in Figure 6. Both results from smartphonepaper device and manual counts are within the expected healthy ranges (dotted square box), which confirms the accuracy of this method for identifying the relative ratios of granulocytes and agranulocytes in the blood. Additionally, the manual and smartphone-paper system counts are very similar to each other. Overall this approach was shown to be remarkably effective for separating WBCs into granulocytes and agranulocytes.

The results from both the differential WBC count as well as total WBC counts demonstrate that the results obtained using the smartphone imaging platform are comparable to those obtained using a benchtop microscope.

\section{CONCLUSION}

While our method does not improve the flow cytometry or other standard WBC counting techniques, the straightforwardness of this approach offers major merits. The three-layered paper device is low cost and easy to fabricate. The device requires only $1-4 \mu \mathrm{L}$ of blood per test which can be obtained from a finger prick. The device also provides on-paper mixing, capture, concentration, and separation, all in an automated, spontaneous manner. The total assay time from finger-prick to data collection is under five minutes. Another unexpected highlight is that WBCs are captured at a predictable rate for $(5 \%, 10 \%$ and $20 \%)$ blood dilutions. Additionally, demonstrating fluorescence imaging and a WBC differential on a paper platform is a first for complete blood count technologies.

We also demonstrated that similar results to those obtained using the benchtop fluorescence microscope can be easily replaced with a commercially available smartphone microscope attachment coupled with an optical filter and a blue light source.

There are a number of areas where the present work could see improvement. For example, occasionally this approach was shown to yield results with a significant degree of error. This could likely be improved by collecting a larger field of view from each sample, reducing the sample variance. Alternatively, a larger field of view could be obtained using an automated stage to collect multiple images and employ image post processing to stitch them together.

Our data processing code also came short of being wholly automated. Therefore, some future work would be required to replace the manually adjusted elements of the code with automated processes before this work could be commercialized or used in a high-throughput manner. Even with these challenges this work presents significant findings and suggests that this device and method could be effectively employed in resource poor settings by untrained personnel.

\section{REFERENCES}

1] B. George-Gay and K. Parker, "Understanding the complete blood count with differential," J. PeriAnesthesia Nurs., vol. 18, no. 2, pp. 96-117, Apr. 2003.

[2] M. C. Walters and H. T. Abelson, "Interpretation of the complete blood count.," Pediatr. Clin. North Am., vol. 43, no. 3, pp. 599-622, Jun. 1996.

[3] P. Lutz and W. Dzik, "Large-volume hemocytometer chamber for 
accurate counting of white cells (WBCs) in WBC-reduced platelets: validation and application for quality control of WBC-reduced platelets prepared by apheresis and filtration," Transfusion, vol. 33, no. 5, pp. 409-412, May 1993.

[4] D. Bodensteiner, "A flow cytometric technique to accurately measure post-filtration white blood cell counts," Transfusion, vol. 29, no. 7, pp. 651-653, Sep. 1989

[5] C. P. Price, "Point of care testing.," $B M J$, vol. 322, no. 7297, pp. 1285-8, May 2001.

[6] U. Hassan et al., "A microfluidic biochip for complete blood cell counts at the point-of-care.," Technology, vol. 3, no. 4, pp. 201-213, Dec. 2015.

[7] D.-H. Kuan, C.-C. Wu, W.-Y. Su, and N.-T. Huang, "A Microfluidic Device for Simultaneous Extraction of Plasma, Red Blood Cells, and On-Chip White Blood Cell Trapping.," Sci. Rep., vol. 8, no. 1, p. 15345 , Oct. 2018.

[8] D. Holmes et al., "Leukocyte analysis and differentiation using high speed microfluidic single cell impedance cytometry," Lab Chip, vol. 9 , no. 20, p. 2881, Oct. 2009.

[9] L. V. Rao, B. A. Ekberg, D. Connor, F. Jakubiak, G. M. Vallaro, and M. Snyder, "Evaluation of a new point of care automated complete blood count (CBC) analyzer in various clinical settings," Clin. Chim. Acta, vol. 389, no. 1-2, pp. 120-125, Mar. 2008.

[10] X. Chen, T. Li, H. Zeng, Z. Hu, and B. Fu, "Numerical and experimental investigation on micromixers with serpentine microchannels," Int. J. Heat Mass Transfer, vol. 98, pp. 131-140, Jul. 2016.

[11] Y. Zhang, J. Bai, H. Wu, and J. Y. Ying, "Trapping cells in paper for white blood cell count," Biosens. Bioelectron., vol. 69, pp. 121127, Jul. 2015

[12] X. Wang, G. Lin, G. Cui, X. Zhou, and G. L. Liu, "White blood cell counting on smartphone paper electrochemical sensor," Biosens. Bioelectron., vol. 90, pp. 549-557, Apr. 2017.

[13] U. M. Jalal, G. J. Jin, and J. S. Shim, "Paper-plastic hybrid microfluidic device for smartphone-based colorimetric analysis of urine," Anal. Chem., vol. 89, no. 24, pp. 13160-13166, Dec. 2017.

[14] X. Chen and J. Shen, "Review of membranes in microfluidics," $J$. Chem. Technol. Biotechnol., vol. 92, no. 2, pp. 271-282, Feb. 2017.

[15] G. Choi and S. Choi, "Cellular flow in paper-based microfluidics," Sensors Actuators B Chem., vol. 237, pp. 1021-1026, Dec. 2016.

[16] R. B. Channon et al., "Rapid flow in multilayer microfluidic paperbased analytical devices," Lab Chip, vol. 18, no. 5, pp. 793-802, Feb. 2018.

[17] M. S. Blumenreich, The White Blood Cell and Differential Count. Butterworths, 1990.

[18] M. R. Melamed, L. R. Adams, A. Zimring, J. G. Murnick, and K. Mayer, "Preliminary Evaluation of Acridine Orange as a Vital Stain for Automated Differential Leukocyte Counts," Am. J. Clin. Pathol., vol. 57, no. 1, pp. 95-102, Jan. 1972.

[19] C. E. Majors, M. Pawlowski, T. Tkaczyk, and R. Richards-Kortum, "Imaging Based System for Performing a White Blood Cell Count and Partial Differential at the Point of Care," in Biomedical Optics 2016, 2016, p. TTu2B.3.

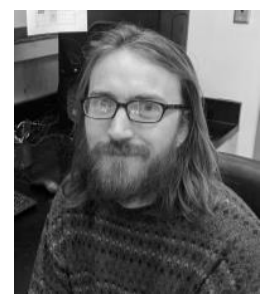

Matthew V. Bills was born in Lander, WY, USA in 1985. He received B.S. degree in biology from The Brigham Young University - Idaho, Rexburg, ID, USA in 2011. He is a Ph.D. candidate in biomedical engineering at the University of Arizona. Since 2017, he has been a graduate research assistant in the University of Arizona Biosensors Lab in Tucson, AZ, USA. His research interests include the development of biosensors for medical monitoring and diagnostics.

Mr. Bills is a recipient of the Biomedical Imaging and Spectroscopy Training Grant from the U.S. National Institutes of Health, grant number T32-EB000809.

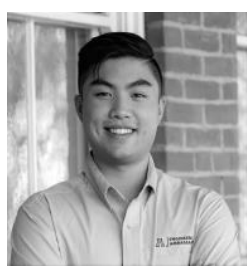

Brandon T. Nguyen is from Prescott, Arizona, USA. He is expected to receive a B.S. degree in biomedical engineering from the University of Arizona in May 2020. Since 2017, he has been an undergraduate research assistant in the University of Arizona Biosensors Lab in Tucson, AZ, USA. His research interest has been in the development of affordable diagnostics.

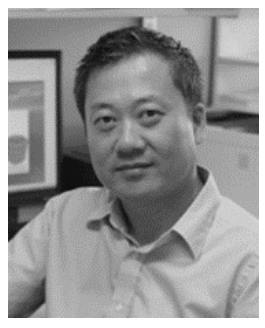

Jeong-Yeol Yoon was born in Seoul, Republic of Korea, and received his B.S., M.S. and Ph.D. degrees in chemical engineering from Yonsei University, Seoul, in 1992, 1994 and 1999, respectively, where he worked primarily on polymer colloids. He received his second Ph.D. degree in biomedical engineering from University of California, Los Angeles, CA, USA in 2004, working on lab-on-a-chip and biomaterials. He joined the faculty at the University of Arizona, Tucson, AZ, USA in August 2004 and currently Professor and Associate Department Head in biomedical engineering department, as well as Professor in biosystems engineering department and chemistry \& biochemistry department. He is currently directing Biosensors Lab. He has published numerous journal articles in the field of biosensors, including IEEE Sensors Journal, Biosensors and Bioelectronics, Science Advances, Advanced Materials, Lab on a Chip, etc. He is also a sole author of Introduction to Biosensors: From Electric Circuits to Immunosensors (currently second edition; New York, NY, Springer, 2016). Dr. Yoon is a member of Institute of Biological Engineering (IBE), Biomedical Engineering Society (BMES), American Society of Agricultural and Biological Engineers (ASABE), and SPIE: The International Society for Optics and Photonics. He was President of IBE in the calendar year 2015. He currently serves as Editor-in-Chief for Journal of Biological Engineering (BMC), and Editorial Board Member for Scientific Reports (Nature Publishing Group). 